\title{
TAXA DE CHUMBO EM AMOSTRA DE VOLUNTARIOS "NÃO EXPOSTOS" HABITANTES DA GRANDE SÃO PAULO - BRASIL
}

\author{
Diogo Pupo Nogueira* \\ Sérgio Colacioppo * \\ José Maria Pacheco de Souza ** \\ Cleide Bernardes Pezza *** \\ Marlene Lopes Assis de Souza * \\ Jorge da Rocha Gomes*
}

\begin{tabular}{l|l}
\hline RSPUB9/458 \\
\hline
\end{tabular}

Nogueira, D. P. et al. Taxa de chumbo em amostra de voluntários "nüo expostos" habitantes da Grande Sáo Palllo. Rev. Saúde públ., S. Paulo, 13: 147-50, 1979.

RESUMO: Através de um espectrofotômetro de absorção atômica foram pesquisados os niveis de chumbo no sangue de 315 voluntários de ambos os sexos (167 do sexo masculino e 148 do feminino) que não revelaram exposição ocupacional a esse metal. Foi encontrado um valor médio de $17,2 \mu \mathrm{g} / 100 \mathrm{ml}$ para o sexo masculino e $14,2 \mu \mathrm{g} / 100 \mathrm{ml}$ para o sexo feminino. Sugere-se a execução de novos estudos que envolvam populaçóes definidas e amostragem probabilistica.

UNITERMOS: Chumbo. Espectrofotometria de absorção atômica.

I N T R O DUCA O

O chumbo é um elemento químico conhecido e utilizado industrialmente desde os povos antigos, e devido à sua larga aplicação é encontrado atualmente bastante disperso no nosso meio ambiente, aumentando sua concentração normal nos organismos vivos.

Um trabalhador exposto profissionalmente a chumbo terá seu nivel elevado no sangue em virtude desta exposição, porém, outro indivíduo não exposto profissionalmente ao metal poderá, igualmente, ter seu nivel sangüineo aumentado em virtude de exposição não ocupacional, através do ar, água e alimentos; desta forma torna-se tarefa difícil determinar se um indivíduo está ou não com níveis elevados de chumbo no sangue em virtude de uma exposição ocupacional ou ambiental.

Alguns autores desenvolveram estudos e apresentaram resultados de concentrações por eles consideradas "normais" de chumbo

* Do Departamento de Saúde Ambiental da Faculdade de Saúde Pública da USP - Av. Dr. Arnaldo, 715 - 01255 - São Paulo, SP - Brasil.

* Do Departamento de Epidemiologia da Faculdade de Saúde Pública da USP - Av. Dr. Arnaldo, 715 - 01255 - São Paulo, SP - Brasil.

*** Da Fundação Centro Nacional de Segurança, Higiene e Medicina do Trabalho - Alameda Barão de Limeira, 539 - 01202 - São Paulo, SP - Brasil. 
Togueira, D. P. et al. Taxa de chumbo en amostra de roluntános "não expostos" habitantes da Grande São Paulo. Rev. Saúde públ., S. Paulo. 13.14i-50, 1979.

no sangue. Goldwater e Hoover,-2 en estudo internacional con residentes de vários paises concluiram que niveis de $15 \mu \mathrm{g} / 100 \mathrm{ml}$ a $40 \mu \mathrm{g} / 100 \mathrm{ml}$ poderiam ser considerados como indicativos de uma exposição ambiental e não ucupacional. No Brasil, tem-se conhecimento do trabalho de Canella ${ }^{2}$, que encontrou em individuos não expostos, o valor médio de $20 \mu \mathrm{g}$ de chumbo por $100 \mathrm{~g}$ de sangue, em uma amostragem de 92 adultos do sexo masculino.

No presente trabalho o objetivo foi o de conhecer a distribuição da concentração de chumbo no sangue en individuos que se declararam não expostos profissionalmente a este elemento químico. Desta forma, os niveis encontrados poderiam ser atribuidos a fatores ambientais, tais como inalação de emanações de veiculos automotores e de fábricas, ingestão de alimentos e água contaminados por chumbo, entre outros.

Em virtude da amostra de indivíduos ser de voluntários de uma população nāo exatamente definida, nāo foram feitas inferências mais gerais e, principalmente, nenhuma tentativa de estabelecer padrões de normalidade de concentração de chumbo no sangue, servindo o presente trabalho para se obter uma icléia geral do problema e, também, como base para uma pesquisa de maior amplitude.

\section{MATERIAL E Metodos}

Foram obtidas amustras de sangue de uma população de trabalhadores de empresas industriais (de produtos alimenticios, de plásticos e de produtos eletrônicos); funcionários de um jornal, estudantes de curso pré-vestibular da Capital de São Paulo; pessoas não portadoras de doença atendidas no Centro de Saúde da Barra Funda; pessoal do Hospital das Clinicas da Faculdade de Medicina da Universidade de São Paulo (USP); e pessoal da Faculdade de Saude Pública da USP. Em relação aos empregados de empresas industriais, o maximo cuidado foi tido no sentido de só se ohter sangue dacueles que se dedicassem a atividades onde comprovadamente não houvesse exposição ocupacional a chumbo. Decidiu-se limitar as idades entre 20 a 69 anos inclusive, tendo sido examinatos, então, o sangue de 148 mulheres e de 167 homens.

As amostras de $5 \mathrm{ml}$ de volume de sangue foram colhidas com seringas plásticas descartáveis, previamente heparinizadas. Após a colheita, as seringas foram fechadas por meio de protetor plástico de agulha e armazenadas sob refrigeração até 0 momento da análise no laboratório.

As amostras de sangue foram analisadas no laboratório de Toxicologia Industrial do Departamento de Saúde Ambiental da Faculdade de Saude Pública da USP, que funciona em convênio com a Fundação Centro Nacional de Segurança, Higiene e Medicina do Trabalho - FUNDACENTRO.

A análise quimica foi feita segundo o método descrito por Hessel : : uma alíquota de $5 \mathrm{ml}$ de sangue é hemolizada com solução de Triton-X-100; o chumbo é complexado com pirrolidin ditiocarbonato de amônio (Eastman No. 9279) e o complexo extraído com iso-butil-metilcetona. O extrato foi analisado por espectrofotometria de absorção atômica, com equipamento Perkin Elmer 360 .

Para cada sexo foram calculadas as concentraçñes média e mediana de chumbo no sangue, a variância e o desvio padrão e obtidas a moda, a amplitude de variação e us valores máximo e mínimo, tendo-se testadu a diferença entre as médias pelo teste $t$ de Student. As distribuições de frequiência foram apresentadas sob a forma de histogramas. Estudou-se, ainda, a forma de distribuição, testando-se a possivel normalidade, através do teste de Kolmogorov-Smirnov, com ajuste segundo Lillefors ${ }^{+}$ (1967).

\section{RESULTADOS E COMENTARIOS}

A Tabela mostra os valores de chumbo encontrados, podendo-se notar yue as amplitudes de variação são praticamente as mesmas nos dois sexos, com valores mini- 
Nogueira, D. P. et al. Taxa de chumbo em amostra de voluntálios "nâo expostos" habitantes da Grande São Paulo. Rev. Saúde puibl,. S. Paulo, 13:147-50, 1979.

mo e máximo tambén extremamente próximos. No sexo masculino a menor concentraçāo observada foi $5 \mu \mathrm{g} / 100 \mathrm{ml}$ e no sexo feminino $3 \mu \mathrm{g} / 100 \mathrm{ml}$; as concentrações maiores foram $44 \mu \mathrm{g} / 100 \mathrm{ml}$ e $45 \mu \mathrm{g}$ na mesma ordem. As variabilidades são tanbém semelhantes, como mostram os desvios padrão de $7,8 \mu \mathrm{g} / 100 \mathrm{ml}$ e $7,1 \mu \mathrm{g} / 100 \mathrm{ml}$.

T A B E L A

Mádia, mediana. mola amphtude de rariação e desrio padrão das concentrações de vhumbo no sangue ( $\mu$ g $100 \mathrm{ml}$ ) em voluntáros residentes na Grande São Paulo, segundo sexo. $197 \pi$.

\begin{tabular}{l|ccc}
\hline & Sexo masculıno & Sexo feminino \\
\hline Média & 17.2 & 14.2 \\
Mediana & 16 & 13 \\
Moda & 10 & 12 \\
Amplitude de rariação & 5 & a 44 & 3 a 45 \\
Desvio padrão & 7.8 & 7.1 \\
\hline Tamanho da amostráa & 167 & 148 \\
Idade média & 35.2 anos & 35.1 anos \\
\hline
\end{tabular}

Ao se comparat os valores médios, verifica-se que o sexo masculino apresenta valor mais alto do yue o sexo feminino, resultado que é acompanhado pelas medianas $(17,2 \mu \mathrm{g} / 100 \mathrm{ml} \times 14,2 \mu \mathrm{g} / 100 \mathrm{ml} \mathrm{e}$ $16 \mu \mathrm{g} / 100 \mathrm{ml} \times 13 \mu \mathrm{g} / 100 \mathrm{ml})$. A diferença

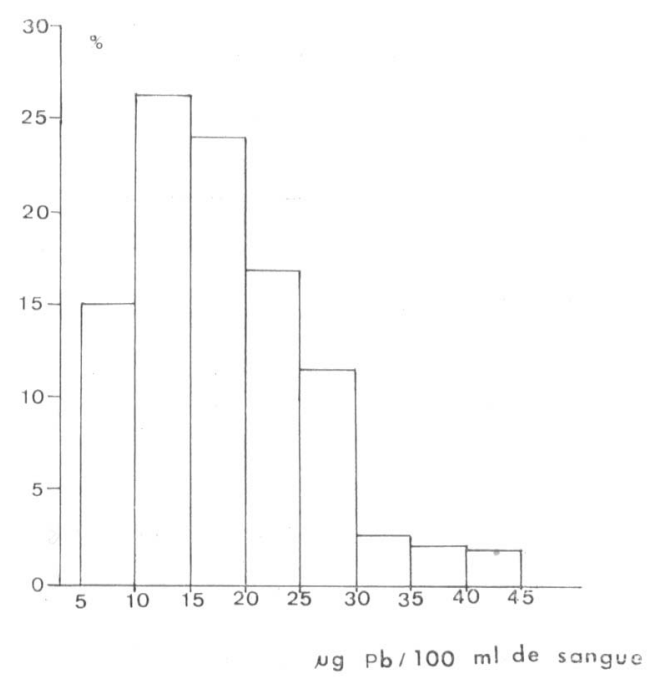

Fig. 1 - Distribuicão de indivíduos do sexo masculino segunto taxa de chumbo no sangue. é estatisticamente significante, a um nivel de $1 \%$ (teste bicaudal), com o valor observado de $t=3,563$.

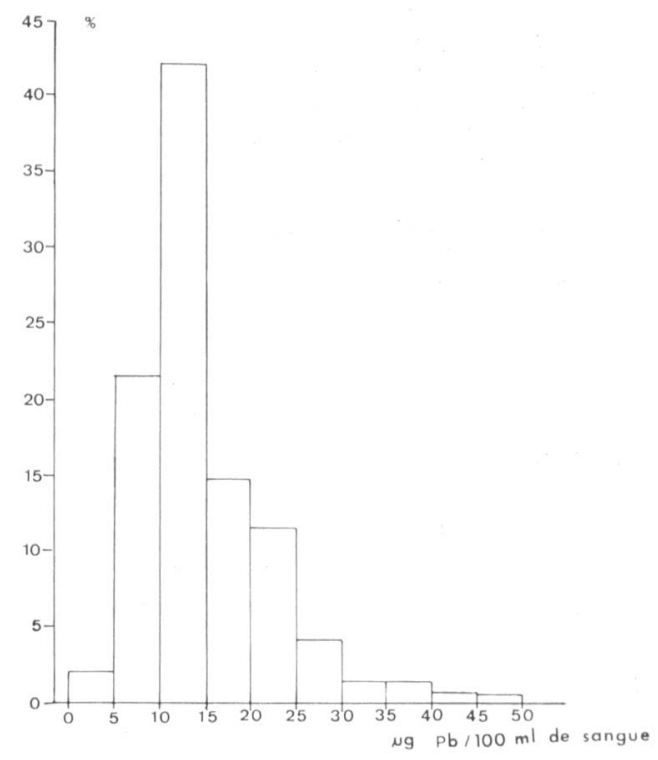

Fig. 2 - Distribuição de indivíduos do sexo feminino, segundo taxa de chumbo no sangue. 
NOGUEIRA, D. P. et al. Taxa de chumbo em amostra de voluntários "não expostos" habitantes da Grande São Paulo. Rev. Saúde públ., S. Paulo, 13:147-50, 1979.

As Figuras 1 e 2 são os histogramas correspondentes às distribuições de freqüência das duas amostras. A distribuição no sexo feminino parece ser mais assimétrica do que aquela do sexo masculino, havendo ainda uma grande concentração de observações na classe $10-15 \mu \mathrm{g} / 100 \mathrm{ml}$, 0 que explicaria estatisticamente a diferença de médias já mencionada. Para a confirmação do presente resultado e explicações epidemiológicas, fazem-se necessários novos estudos que envolvam populaçōes bem definidas e amostragem probabilística.

As formas das distribuições foram também testadas para normalidade. Para um nivel de $10 \%$ de significância, foi possivel aceitar-se a normalidade para o sexo masculino $(p>0,20)$, mas não para o sexo feminino $(p<0,01)$. Decidiu-se, então, verificar se a transformação logarítmica satisfazia aquela condição; ainda a nivel de $10 \%$ de significância, a variável "logaritmo na base de 10 da concentração de chumbo" foi aceita como normal, para ambos os sexos, com os valores $0,20>p>0,05$ para o sexo masculino e $0,15>p>0,10$ para o sexo feminino.

\section{AGRADECIMENTOS}

Aos técnicos Maria Lusia Rodrigues Pereira, Maria Helena Callera Pedrosa e Juan Canet Font, da Fundação Centro Nacional de Segurança, Higiene e Medicina do Trabalho, pela colaboração na colheita de amostras de sangue e no auxílio às operações de análise.

NogueIRA, D. P. et al. /The level of lead in blood samples from non-exposed volunteer residents of Great S. Paulo (Brazil)]. Rev. Saúde públ., S. Paulo, $13: 147-50,1979$.

ABSTRACT: The level of lead in the blood of 315 volunteers (167 male and 148 female) who had not been exposed professionally to the metal, was measured by the atomic absortion spectrophotometer method. The average value was $17.2 \mu \mathrm{g} / 100 \mathrm{ml}$ in the males and $14.2 \mu \mathrm{g} / 100 \mathrm{ml}$ in the females. It is suggested that new studies should be carried out, using a definite population and a probabilistic sampling.

UNITERMS: Lead. Spectrophotometry, atomic absorption.

\section{REFERENCIAS BIBLIOGRAFICAS}

1. CANElla. D. J. Concentração de chumbo no sangue de trabalhadores industriais nāo expostos ao metal. In: Congresso Americano de Medicina do Trabalho, São Paulo, 1964. Anais. São Paulo. ABPA, 1964, p. 300-1.

2 GOLDWATER, J, \& HOOVER, W. An international study of "normal" levels of lead in blood and urine. Arch. environm. Hith, 15:60-3, 1967.
3. HESSEL, D. W. A simple and rapid quantitative determination of lead in blood. At. Absor. Newsl., 7:55-6, 1968.

4. LILLEFORS, H. W. On the KolmogorovSmirnov test for normality with mean and variance unknown. $J$. Amer. statist. Ass., 62:399-402, 1967.

Recebido para publicasado em 05/01/197y Aprovado nara publicagāo em 22/03/197\% 


\section{ERRATA}

\section{REVISTA DE SAÚDE PÚBLICA, 13 (2), 1979.}

p.150, segundo parágrafo, 12a linha.

Onde se lê: ..., com os valores $0,20>p>0,05$ para o sexo masculino...

Leia-se: ..., com os valores $0,20>p>0,15$ para o sexo masculino...

p.113-Autores

Onde se lê: Tadeu de Assis

Leia-se: Arthur Tadeu de Assis 
O arquivo disponível sofreu correções conforme ERRATA publicada no Volume 13 Número 3 da revista. 